\title{
Accurate timing of gamma rays with high-rate Resistive Plate Chambers
}

\author{
L. Lopes ${ }^{\mathrm{a}}$, A. Pereira ${ }^{\mathrm{a}}$, P. Fonte ${ }^{\mathrm{a}, \mathrm{c}}$, R. Ferreira Marques ${ }^{\mathrm{a}, \mathrm{b}, *}$ \\ ${ }^{a}$ Laboratório de Instrumentação e Física Experimental de Partículas, 3004-516 Coimbra, Portugal \\ ${ }^{\mathrm{b}}$ Departamento de Física, Faculdade de Ciências e Tecnologia da Universidade de Coimbra, 3004-516 Coimbra, Portugal \\ ${ }^{\mathrm{c}}$ Instituto Superior de Engenharia de Coimbra, 3030-199 Coimbra, Portugal
}

Available online 20 November 2006

\begin{abstract}
The counting rate capability of RPCs is strongly conditioned by the availability of suitable resistive materials for the electrodes. For time-of-flight measurements in heavy-ion high-energy physics, the extension of the counting rate capabilities achievable with glass electrodes, around $2 \mathrm{kHz} / \mathrm{cm}^{2}$, to much higher values is of fundamental importance. To address this issue we developed single-gap tRPCs with electrodes made from metal and from a commercially available ceramic material free of charge-depletion effects. Tests performed with $511 \mathrm{keV}$ photons yielded a time resolution around $90 \mathrm{ps} \sigma$. Neither the time resolution nor the efficiency have been affected by background rates of up to $20 \mathrm{kHz} / \mathrm{cm}^{2}$ produced with photons from an X-ray generator. The present result establishes the practical feasibility of accurate timing measurements with RPCs at rates up to $20 \mathrm{kHz} / \mathrm{cm}^{2}$, while keeping a time resolution below $100 \mathrm{ps} \sigma$.

(C) 2006 Elsevier B.V. All rights reserved.
\end{abstract}

Keywords: RPC; TOF; High-rate

\section{Introduction}

Timing resistive plate chambers (tRPCs) are planar gaseous detectors made with resistive electrodes that form very thin and accurately defined gas gaps. Such detectors are known to deliver timing accuracies around $50 \mathrm{ps} \sigma$ for minimum ionizing particles [1]. These excellent timing characteristics, together with their low cost and the possible coverage of large areas, made such detectors valuable instruments for time of flight (TOF) systems used in particle identification, particularly in heavy ion highenergy physics. In addition, the accurate timing is achievable without compromising other intrinsic features of the RPCs, in particular the good position resolution that is so important for many other applications.

In view of future high intensity machines, it is of fundamental importance that the present counting rate capability of tRPCs is very much improved. The maximum counting rate achievable, at present around $2 \mathrm{kHz} / \mathrm{cm}^{2}$ with glass electrodes, is limited by the lack of other electrode materials that, besides of having the necessary mechanical properties, also have the adequate resistivity.

\footnotetext{
${ }^{*}$ Corresponding author.

E-mail address: rui@lipc.fis.uc.pt (R. Ferreira Marques).
}

Upon having investigated a few other candidate materials, we recently constructed and tested two single-gap tRPCs with a metallic cathode and an anode made from a commercially available ceramic material with a resistivity of $10^{9} \Omega \mathrm{cm}$ [2].

The results reported below show that the time resolution remains essentially unchanged up to a counting rate one order of magnitude higher than the above mentioned limit, without any measurable rate-induced loss of efficiency. The ceramic material used is free of the annoying chargedepletion effects that prevent the use of other materials, such as slightly conductive polymers [3]. Thus, the present result establishes the practical feasibility of accurate timing measurements with tRPCs at rates at least one order of magnitude higher that previously achieved.

\section{Experimental system}

The detectors, with an active area of $9 \mathrm{~cm}^{2}$, had stainless steel cathodes and ceramic anodes, defining a single $0.3 \mathrm{~mm}$ wide gas gap (Fig. 1). The anodes were made out of a commercially available ceramic material with measured resistivity of $10^{9} \Omega \mathrm{cm}$ and free of charge-depletion effects [2]. The electrodes had the active surfaces lapped and 


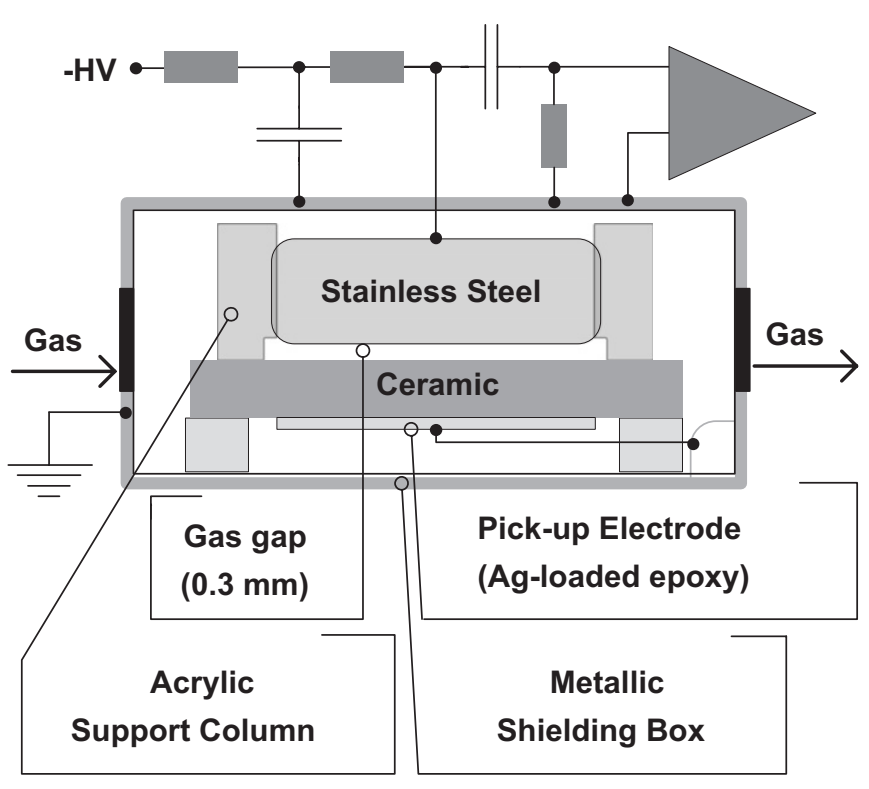

Fig. 1. Schematic view of the detector structure.

polished and their smoothed edges were externally glued to support columns [4]. Electrical contact with the ceramic was made via a uniform layer of silver-loaded epoxy glue [3].

The assembly was made in a clean room, under strict clean conditions, which are essential to avoid permanent discharges.

Each counter was enclosed in a metallic shielding box see (Fig. 1) and the assembly placed in a gas-tight metallic enclosure. A continuous gas flow of $85 \%$ of tetrafluorethane $\left(\mathrm{C}_{2} \mathrm{H}_{2} \mathrm{~F}_{4}\right), 10 \%$ of sulfur hexafluoride $\left(\mathrm{SF}_{6}\right)$ and $5 \%$ of isobutane $\left(\mathrm{i}-\mathrm{C}_{4} \mathrm{H}_{10}\right)$ was piped into the shielding boxes through dust filters [3]. A schematic view of the setup is shown in Fig. 2.

Both chambers produced very similar currents when irradiated, confirming the precise gap spacing. The dark count rates were typically below $1 \mathrm{~Hz} \mathrm{~cm}^{-2}$.

Chamber signals were sensed by a $3 \mathrm{GHz}$ bandwidth, gain 10 , preamplifier [5] and digitized by a $1 \mathrm{GHz}$ bandwidth, $10 \mathrm{GS} / \mathrm{s}$ TEK000 oscilloscope. Signal analysis was done offline by software.

Time resolution tests were performed by irradiating the chambers with pairs of $511 \mathrm{keV}$ photons emitted by a 0.8 $\mathrm{mCi}{ }^{22} \mathrm{Na}$ source placed between them. Higher rate in the test chamber (background) was reached with the help of an $\mathrm{X}$-ray generator, whose photon spectrum peaks at $\sim 30 \mathrm{keV}$. The time resolution $(\sigma)$ of the high rate chamber was estimated by quadratic subtraction of the resolution of the reference chamber (kept at low rate) from the measured time difference spectrum. The resolution of the reference chamber was obtained by measuring the time resolution with the ${ }^{22} \mathrm{Na}$ source in a symmetric situation and assuming that both chambers had identical response functions.

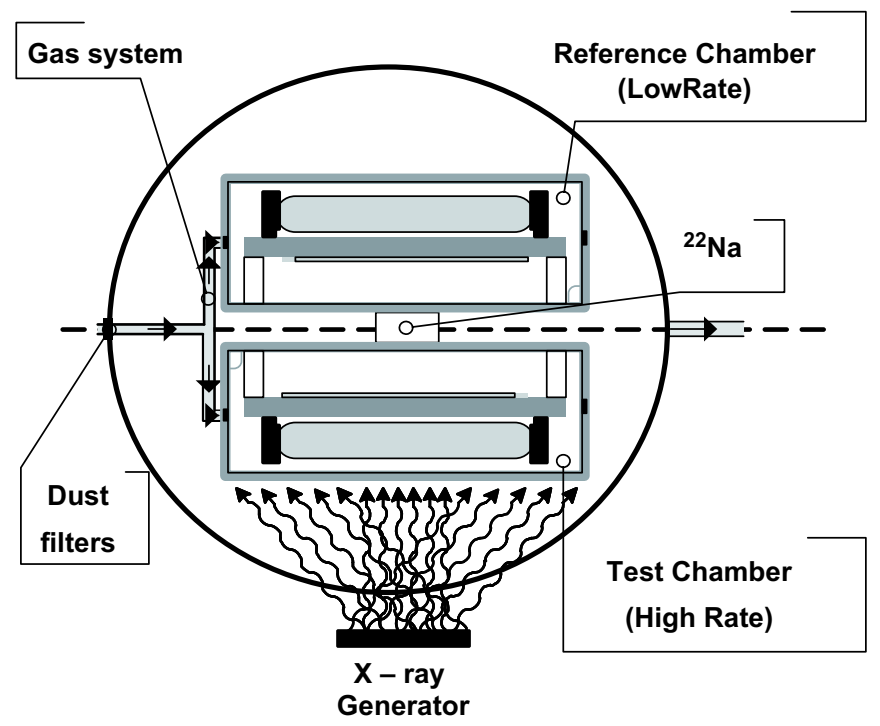

Fig. 2. Schematic representation of the experimental setup. Coincidence events were generated by pairs of photons from a ${ }^{22} \mathrm{Na}$ source. Higher rates in the test chamber were obtained with an X-ray generator which provides a photon spectrum peaking at $\sim 30 \mathrm{keV}$.

\section{Current and counting rate}

The operating current and the counting rate are two of the most important variables for such a kind of detector. Understanding the behaviour of both is essential. Fig. 3(a) shows the counting rate as function of the X-ray anode current, proportional to the incident photon flux. For a threshold of $10 \mathrm{mV}$, the counting rate for any of the three different applied voltages $(2500,2600$ and $2700 \mathrm{~V})$ shows a linear dependence on the incident photon flux within a few percent deviation (Fig. 3b). Thus, we can conclude that, within this margin, the chamber doesn't lose efficiency from 1 to $25 \mathrm{kHz} \mathrm{cm}^{-2}$.

Even at the highest counting rate we could operate at all applied voltages in a streamer free regime.

The operation current as a function of the incident particle flux is shown in Fig. 3(b). It is clear that the behavior, although non linear, is far from saturation. Indeed, following the Ohm law, the applied voltage in a planar is detector given by

$V_{\text {ap }}=V_{\text {eff }}+i R$,

where $V_{\text {eff }}$ is the effective voltage across the gap, $i$ is the chamber current and $R$ the total resistance seen by the current, which is mostly defined by the volume resistivity of the electrodes.

For a given current, electrodes with a lower resistivity present smaller drop of potential, iR, and most of the applied voltage goes into effective gap voltage. As the amplification of the initial charges has an exponential dependence on the effective voltage, this causes an increase of the avalanche mean charge, followed by an increase of the operation current, when compared to detectors with higher resistivity electrodes (e.g., glass and bakelite). 
a

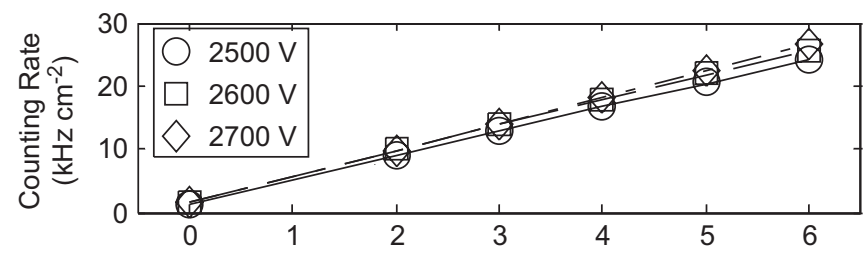

b

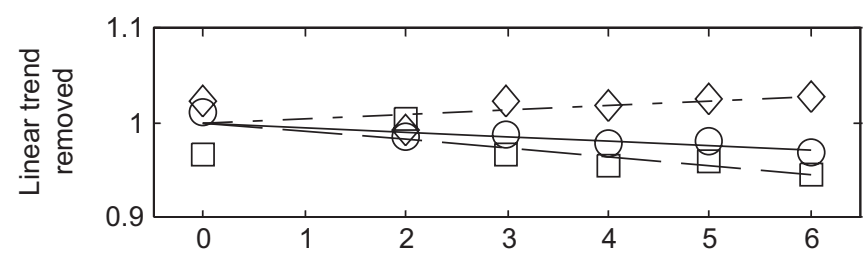

C

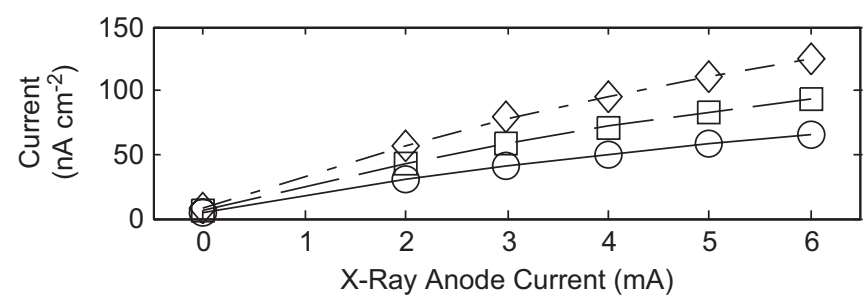

Fig. 3. (a) Counting rate as a function of X-ray anode current grows linearly with the incident photon flux, for all applied voltages. The lines correspond to an adjusted quadratic function. (b) Same as (a), but normalized to the linear part of the fit to evidence departures from linearity. (c) Current as function of the X-ray anode current (proportional to the incident photon flux). In contrast with the counting rate behavior, here we observe a moderate non-linearity with the incident photon flux.

\section{Time resolution}

For the fit to the time spectra, we use a Gaussian (real coincidences) plus a symmetrical exponential (background). The use of X-ray photons increases the rate of random coincidences without affecting the time resolution. This is visible in Fig. 4, where the time spectrum between the two chambers is shown. The time resolution $(\sigma)$ of the high rate chamber is estimated by quadratic subtraction of the resolution of the reference chamber ( $85 \mathrm{ps}$ ) from the measured time difference spectrum, 123 ps.

As shown in Fig. 5, the time resolution remained essentially unchanged, at around $90 \mathrm{ps} \sigma$, from 1 to $20 \mathrm{kHz} \mathrm{cm}^{-2}$. It should be noted that previous experience with timing RPCs in particle beams and with annihilation photons has shown that, while single-gap counters may reach a time resolution of $60 \mathrm{ps} \sigma$ in particle beams [6], similar counters irradiated with $511 \mathrm{keV}$ photon pairs reach only about $90 \mathrm{ps} \sigma$. This fact is ascribed to the larger statistical variance of the primary charge resulting from the gamma interaction (see [7]).

\section{Conclusion}

The present results establish the basic feasibility of timing measurements with RPCs at rates up to $20 \mathrm{kHz} \mathrm{cm}^{-2}$

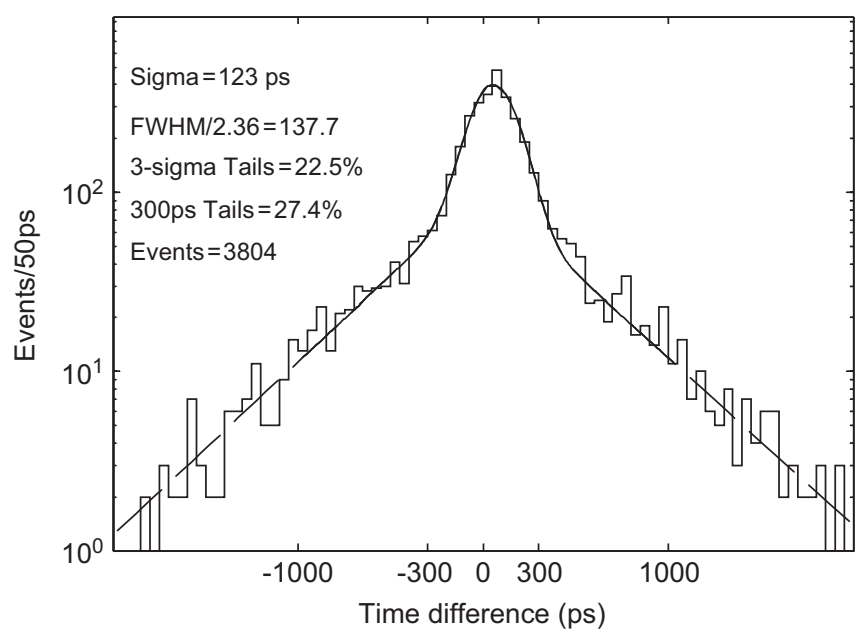

Fig. 4. Time spectrum obtained at $10 \mathrm{kHzcm}^{-2}$. Although the photons from the X-ray generator produce many random coincidences, the time resolution remains unaffected.

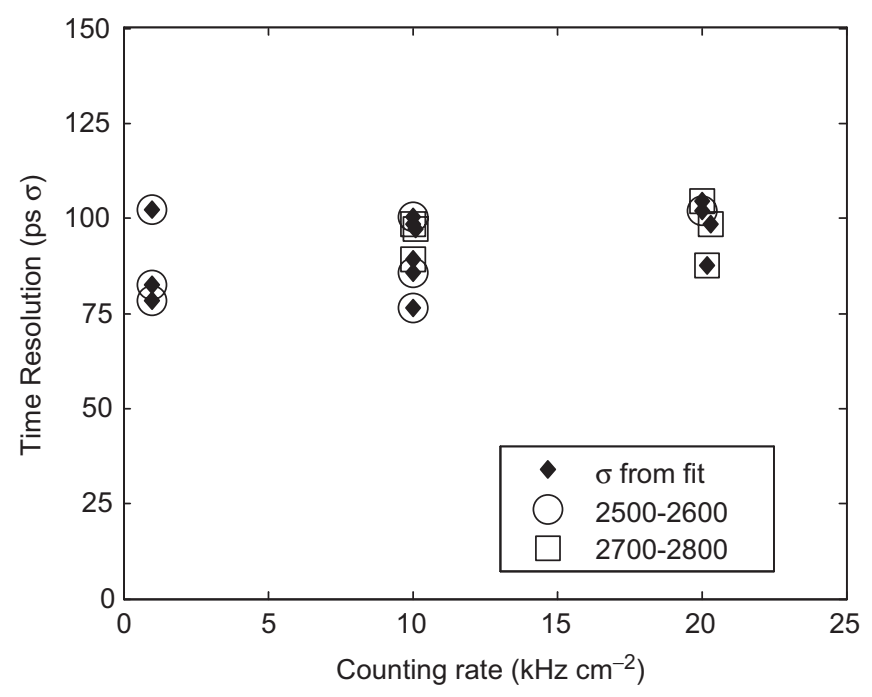

Fig. 5. Time resolution as a function of the counting rate. Within the spread of the points, indicative of the measurement errors, the time resolution remains essentially unchanged (around $90 \mathrm{ps} \sigma$ ) between 1 and $20 \mathrm{kHz} \mathrm{cm}^{-2}$. The horizontal spread, in turn, is due to the different working voltages at which data have been taken.

while keeping a good time resolution (below $100 \mathrm{ps} \sigma$, for gamma ray pairs).

This represents an improvement of over one order of magnitude in counting rate as compared to the highest rates reported so far.

It should be stressed that we have proven that small-area timing RPCs can be made with a commercially available ceramic material.

In addition the use of such ceramics also solves the charge depletion problem that was identified with other candidate materials of adequate resistivity, as was the case of some plastics previously investigated [3]. 
As the counting rate limit of this kind of electrode material was not yet reached in the present studies, further work remains to be done.

\section{Acknowledgements}

This work was partly financed by "Fundação para a Ciência e Tecnologia" projects POCTI/FP/FNU/ 50171/ 2003, POCI/FP/63411/2005, FEDER, the EU 6th Framework Program via contract RII3 CT-2003-506078 and the program INTAS 03-54-3891.

\section{References}

[1] P. Fonte, A. Smirnitski, M.C.S. Williams, Nucl. Instr. and Meth. A 443 (2000) 201.

[2] Controlled resistivity alumina, A1940CD. Morgan Advanced Ceramics, 26 Madison Road, Fairfield, NJ 07004, USA.

[3] L. Lopes, R. Ferreira Marques, P. Fonte, A. Pereira, V. Peskov, A. Policarpo, Nucl. Instr. and Meth. A 533 (2004) 69.

[4] Ch. Finck, et al., Proceedings of the XXXIX International Winter Meeting on Nuclear Physics, Bormio, 2001.

[5] A. Blanco, et al., IEEE Trans. Nucl. Sci. 48 (4) (2001) 1249.

[6] P. Fonte, et al., Nucl. Instr. and Meth. A 508 (2003) 70.

[7] A. Mangiarotti, et al., Nucl. Instr. and Meth. A 533 (2004) 16. 